\title{
Evaluation of the Metabolic Profile of Arabica Coffee via NMR in Relation to the Time and Temperature of the Roasting Procedure
}

\author{
Roger P. Alves, ${ }^{\circledR *, a}$ Nelson R. Antoniosi Filho, ${ }^{\circledR a}$ Luciano M. Lião ${ }^{\circledR b}$ and Igor S. Flores ${ }^{\circledR b, c}$ \\ ${ }^{a}$ Laboratório de Métodos de Extração e Separação, Universidade Federal de Goiás, \\ Campus Samambaia, 74045-155 Goiânia-GO, Brazil \\ ${ }^{b}$ Laboratório de Ressonância Magnética Nuclear, Universidade Federal de Goiás, \\ Campus Samambaia, 74690-900 Goiânia-GO, Brazil \\ 'Instituto Federal de Educação, Ciência e Tecnologia de Goiás, Campus Inhumas, \\ 75402-556 Inhumas-GO, Brazil
}

\begin{abstract}
Coffee is one of the most popular and consumed products in the world, with high nutritional value and economic importance. However, some factors can change the organoleptic properties of a coffee species, without causing significant damage such as loss of important components. The present study evaluated the chemical profile, via nuclear magnetic resonance (NMR), of the main biological properties and substances of the drink, verifying similarities in the composition of different types of arabica coffee made in different conditions, such as the roasting time and temperature. The main components were identified, using information from the literature and a database, and compared with the experimental data of $1 \mathrm{D}$ and $2 \mathrm{D}{ }^{1} \mathrm{H}$ NMR. The spectral data were analyzed and grouped via principal component analysis (PCA) using the Bruker Amix 3.9.14 software. ${ }^{1} \mathrm{H}$ NMR was able to monitor the roasting process and qualify the intact bean and chemical profile of the coffee according to the roasting conditions. Due to the importance of the monitored components, the coffee species analyzed can be identified, along with the appearance of unwanted or adulterating compounds that are normally added to the product to reduce the cost of commercialization.
\end{abstract}

Keywords: Coffea arabica, NMR, temperature, roasting time, PCA

\section{Introduction}

Coffee is one of the most consumed products in the world, especially in the Western world. The high nutritional value and economic importance of this commodity ${ }^{1}$ has sparked the interest of researchers and consumers in relation to its quality, chemical composition, and beneficial effects on human health. ${ }^{2}$ The interest on the part of producers has grown substantially due to the increased consumption of coffee in the last four decades. ${ }^{3}$ Numerous species of coffee have been cataloged, but only three species (Coffea arabica, Coffea canephora, and Coffea liberica) are cultivated in large quantities for the consumer market. ${ }^{4}$ Each species is unique peculiarity in relation to the planting method, presence of defects, soil type, chemical composition of the fruit, climate conditions for cultivation, altitude, type of processing, genetic variation, drying, and storage. ${ }^{5-7}$

*e-mail: rogerokuringa@hotmail.com
In addition, different microorganisms that act in the fermentation process of beans can modify the taste, acidity, shape, and aroma of the product. ${ }^{8}$

Other factors, including the control of the roasting time, can alter the organoleptic properties of a coffee species, maintaining the quality of the drink without losing important characteristics. ${ }^{9}, 10$ The roasting process is essential for the production of bioactive substances that can benefit human health, ${ }^{11}$ such as trigonelline, ${ }^{10,12}$ caffeine, ${ }^{13,14}$ formic acid,,${ }^{15,16}$ and chlorogenic acids. ${ }^{16,17}$ The main biological functions of these substances include inhibiting the production of leukotrienes, stimulating the metabolism and the central nervous system, and increasing cognition, in addition to antioxidant, anticarcinogenic, and antidepressant effects. ${ }^{18}$ For almost a century, different scientific studies have analyzed the complex composition in the coffee aroma, which contains numerous volatile substances from thermal reactions (Maillard and Strecker) that occur through the roasting process, ${ }^{19,20}$ to produce 
different types of commercial coffees by varying only the physical conditions, such as roasting time and temperature. ${ }^{21}$

Recently, several techniques have been applied to detect coffee compounds including chromatographic and spectroscopic techniques, both in isolation and in combination, such as infrared and near infrared spectroscopy, (FTIR, FT-NIR), ${ }^{22-24}$ high performance liquid chromatography (HPLC), ${ }^{25-29}$ high-resolution gas chromatography (HRGC), 27,30,31 nuclear magnetic resonance (NMR), ${ }^{32-34}$ and gas chromatography coupled to mass spectrometry (GC-MS). ${ }^{35-38}$

However, the advantage of NMR is that it does not require previous treatment of the sample (separation and purification) like many other techniques. In addition, a detailed investigation of only one hydrogen spectrum provides information on the chemical composition and structure of the main constituents present in the coffee. Some studies report the use of NMR to identify different chemical composition between robust and arabica species, ${ }^{39}$ and to determine adulterants added to reduce production costs, but did not relate the use of this technique to the physical conditions that provide good quality and less preparation time..$^{40,41}$

Currently, the literature only speculates about product decomposition during roasting or the reaction that leads to its degradation. Acrylamide is one of the products formed during coffee roasting ${ }^{42}$ by the Maillard thermal reaction, an important route that comprises the reaction between reducing sugars. ${ }^{43}$ Several review papers published in the literature ${ }^{31,42}$ on coffee NMR do not include statistical treatment such as principal component analysis (PCA) and hierarchical cluster analysis (HCA) to identify and group of organic compounds of interest. Data processing is very useful to help identify different coffee species (canephora, liberica, or arabica). ${ }^{31}$

In this study, we developed a condition to guarantee the quality of the product, optimizing the process with a small temperature rise and a drastic reduction in the roasting time of the grains. For this purpose, ${ }^{1} \mathrm{H}$ NMR spectral profiles compare shorter (up to $4 \mathrm{~min}$ ) and longer roasting times (up to $16 \mathrm{~min}$ ), which are commonly presented in the literature ${ }^{21,44}$ and indicated by coffee masters ${ }^{45}$ and professionals accredited by the Brazilian Coffee Industry Association (ABIC). Such evaluation of the organoleptic characteristics after the roasting process is highly desirable. The identification of chemical composition via ${ }^{1} \mathrm{H}$ NMR, through a rapid analysis, without previous treatment or derivatization, can verify criteria such as the influence of temperature and the roasting time on the quality of coffee.

\section{Experimental}

\section{Roasting process}

The coffee samples were generated at the School of Agronomy at the Federal University of Goiás (EA/UFG) located on the Campus Samambaia, in the city of Goiânia$\mathrm{GO}$, where the three roasting tests were carried out using a Copacabana ${ }^{\circledR}$ roaster, model T-10 with burner ZA1 and TFI flame controller. Two types of arabica beans were roasted, high and low quality properly ground (type 1 and type 2 , respectively), according to criteria pointed out by a coffee master (EA/UFG), such as the size of the bean, its color, the fragrance of the ground coffee, and the number of defective beans under different physical conditions of time and temperature. These same characteristics are used by the ABIC to evaluate the overall quality of the product on a scale of 0 to 10 . This classification, which is unique in the world, helps consumers to decide which quality of coffee they want to consume. ${ }^{46}$

Table 1 indicates the specifications of the sizes purchased, as well as the time when they were roasted and their temperatures.

Table 1. Type 1 and type 2 samples at different roasting times and temperatures

\begin{tabular}{lcccc}
\hline Roasting & Sample & $\begin{array}{c}\text { Roast } \\
\text { time / min }\end{array}$ & $\begin{array}{c}\text { Temperature / } \\
{ }^{\circ} \mathrm{C}\end{array}$ & Quality (type) \\
\hline \multirow{4}{*}{ Test 1 } & 1 & 16 & 180 & type 1 \\
& 2 & 14 & 180 & type 1 \\
& 3 & 12 & 180 & type 1 \\
& 4 & 10 & 180 & type 1 \\
& 5 & 8 & 180 & type 1 \\
& 6 & 6 & 180 & type 1 \\
& 7 & 4 & 180 & type 1 \\
& 8 & 2 & 180 & type 1 \\
& 9 & 0 & - & type 1 \\
\hline \multirow{3}{*}{ Test 2 } & 10 & 14 & 180 & type 2 \\
& 11 & 12 & 180 & type 2 \\
& 12 & 10 & 180 & type 2 \\
& 13 & 6 & 180 & type 2 \\
& 14 & 4 & 180 & type 2 \\
& 15 & 2 & 180 & type 2 \\
& 16 & 0 & - & type 2 \\
\hline \multirow{4}{*}{ Test 3 } & 17 & 4 & 200 & type 2 \\
& 18 & 2 & 200 & type 2 \\
& 19 & 1 & 200 & type 2 \\
& 20 & 0 & - & type 2 \\
\hline
\end{tabular}

The variables evaluated in the roasting tests were temperature, as it represents the energy expenditure involved in the process; the time used to roast the beans; 
and the type of roasted coffee bean, according to the size of the bean, the fragrance of the ground powder and the number of defective beans. The test configurations were defined by the coffee master and in accordance with works found in the literature. ${ }^{38,39,44}$

Figure 1 illustrates the different matrices obtained according to the different roasting times for arabica coffee beans classified as type 1 , referring to higher quality beans (right), and type 2, referring to lower quality beans (left), identified and classified by a coffee master duly accredited by the ABIC.

Tetradeuterated sodium trimethylsilylpropionate (TMSP- $d_{4}$, Sigma-Aldrich, Saint Louis, USA) was used as a reference compound for the relative integration of signals in deuterated water solvent $\left(\mathrm{D}_{2} \mathrm{O}\right.$, Sigma-Aldrich, Saint Louis, USA). The solvent $\mathrm{D}_{2} \mathrm{O}$ was chosen to evaluate the chemical profile of the drink, as it is consumed. The coffee samples ground in the Cadence ${ }^{\circledR}$ coffee grinder were weighed on a Shimadzu ${ }^{\odot}$ analytical scale with a mass of $0.100 \mathrm{~g}$, and subsequently diluted in $0.800 \mu \mathrm{L}$ of $\mathrm{D}_{2} \mathrm{O}$ with $0.1 \%$ TMSP- $d_{4}$. This mixture was taken to the 1800 Branson ${ }^{\circledR}$ Sonicator, in which a cold extraction took place for 20 min to check all the components in the coffee bean, without promoting any type of alteration by thermolabile components or loss of volatile components by evaporation. Subsequently, filtration with cotton was done to remove the remaining material in the suspension. This heterogeneous material went to the EBA21 Hettich ${ }^{\odot}$ centrifuge for $10 \mathrm{~min}$ and 7,000 rpm, from which the supernatant was removed. A $50 \mu \mathrm{L}$ aliquot of the extract was diluted in $550 \mu \mathrm{L}$ of the same solvent, sending prepared sample for ${ }^{1} \mathrm{H}$ NMR analysis.

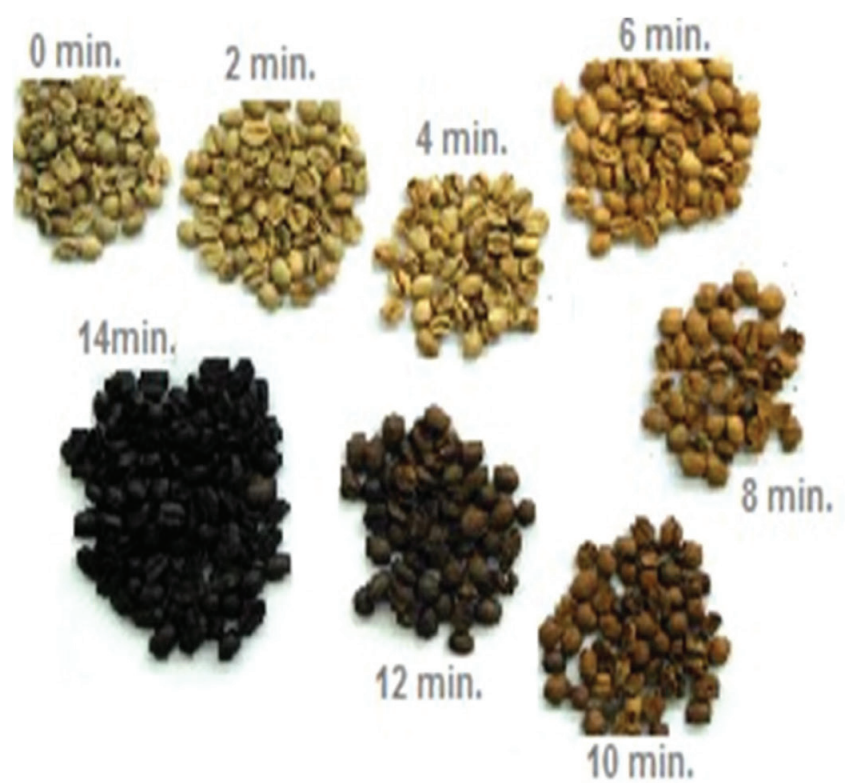

The signal suppression noesyprld sequence was used to suppress the signal strength of the solvent. Acquisition parameters were also established, including acquisition (4s) and relaxation ( $6 \mathrm{~s}$ ) times, as well as processing parameters such as zero filling and line-broadening. A receiver gain of 203 was used, with 128 cycles, and a mixing time of $1.5 \mathrm{~ms}$ with 64 scans. As a result, less equipment time was used without losing the sensitivity and quality, in addition to maintaining the appropriate resolution for our objectives.

The identification of the main compounds was performed through information contained in the literature ${ }^{32,47}$ and access to the database available to our research group. These were compared with experimental $1 \mathrm{D}$ and $2 \mathrm{D}{ }^{1} \mathrm{H}$ NMR data. All experiments were performed in Bruker Avance III $500 \mathrm{MHz}$ at $11.75 \mathrm{~T}$ equipment, with triple resonance broad band (TBI) probe, located in the Nuclear Magnetic Resonance laboratory of the Institute of Chemistry at the UFG (LabRMN-IQ/ UFG).

\section{Commercial coffees}

Table 2 describes information from the samples purchased in Goiânia-GO, Brazil. The type designation indirectly indicates the time the coffee was roasted, with the extra strong type representing the longer roasting time, while the traditional type refers to the shorter roasting time.

\section{Chemometric analyzes}

The chemometric analyses were carried out using the Amix 3.9.14 software (Bruker, Billerica, USA). Thus, the spectral regions referring to the $\mathrm{D}_{2} \mathrm{O}$ signal, the reference

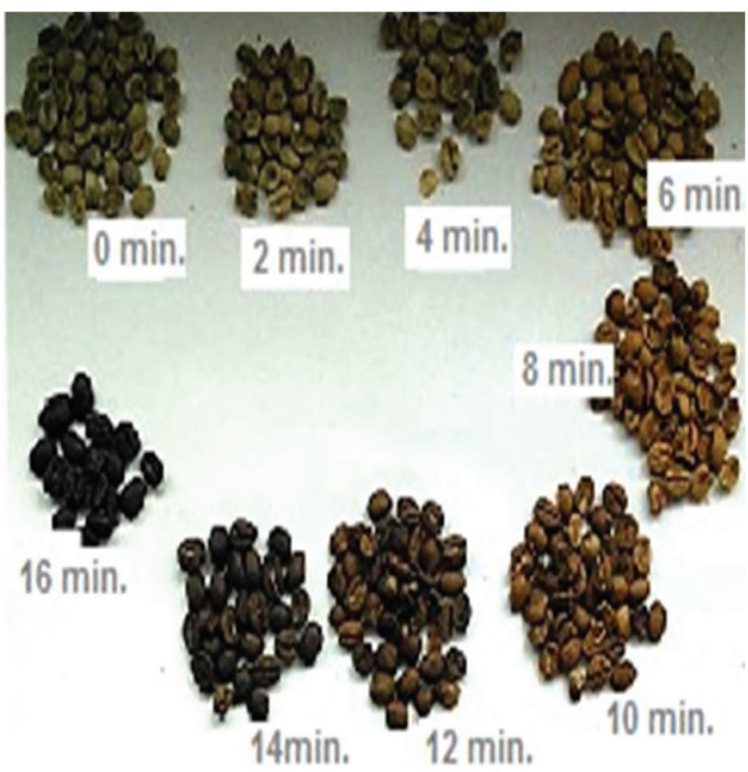

Figure 1. Roasted and unground coffee beans as described in test 2 (left) and test 1 (right). 
Table 2. Description of commercial samples of ground and roasted coffee vacuum packed or pad packed coffee

\begin{tabular}{lclc}
\hline Sample number & Coffee style & Species & Packing \\
\hline 1 & traditional & Arabica & vacuum \\
2 & traditional & Arabica & vacuum \\
3 & uninformed & Arabica & pad \\
4 & traditional & Arabica & pad \\
5 & dark roasting & Arabica & pad \\
6 & extra strong & Arabica & pad \\
7 & extra strong & Arabica & vacuum \\
8 & extra strong & Arabica & vacuum \\
9 & extra strong & Arabica & pad \\
\hline
\end{tabular}

signal TMSP- $d_{4}$, and other regions that contain only spectral noise were removed from the data matrix. The baseline and phase of the spectra were corrected manually using the Topspin software from Bruker. In addition, the $0.5 \mathrm{~Hz}$ line-broadening feature was used to minimize spectral noise by improving the signal-to-noise ratio for each sample analyzed. The PCA was performed using the sum of the absolute intensities with the total scale of intensities and buckets of $0.01 \mathrm{ppm}$.

\section{Results and Discussion}

The coffee roasting process plays a decisive role in the formation of the chemical and organoleptic characteristics of the final product. In the present study, the chemical profile of coffee beans subjected to roasting was analyzed under physical conditions that follow the parameters commonly used by producing industries and by coffee masters, such as roasting time and temperature, to reduce costs in their processing and production.

The roasting times used here were in agreement with other research, ${ }^{21,35}$ and were $14 \mathrm{~min}$ at $180{ }^{\circ} \mathrm{C}$ for type 1 , and 12 min at $180^{\circ} \mathrm{C}$ for type 2 . After optimizing the process for the shortest heating time, the 4 min was used at $200{ }^{\circ} \mathrm{C}$ for type 2 (Figure 2). This information was useful for checking the metabolic profile to achieve a more accepted market condition. The spectra have very similar profiles, which indicate the similarity in quality and type of species, with emphasis on a greater quantity of carbohydrates preserved with the heating time of $4 \mathrm{~min}$ (Figure 2). The spectral analysis of ${ }^{1} \mathrm{H} \mathrm{NMR}$ indicated that the roasting time can be reduced without compromising the chemical composition, because the concentrations of essential substances do not degrade the proposed physical conditions, guaranteeing a spectral similarity, thus maintaining the quality and the sensory properties of the drink. The roasting properties of coffee mostly depend on the amount of heat transferred to the beans of the product during roasting and the time it takes this process. ${ }^{42}$

Figure 3 shows the signals of the compounds of interest identified in the ${ }^{1} \mathrm{H}$ NMR spectrum for test 1 . Figure 4 illustrates the oscillation of these components as a function of time for the same test at constant temperature. Only the signals from the unobstructed chemical groups were integrated via the TMSP- $d_{4}$ spectral

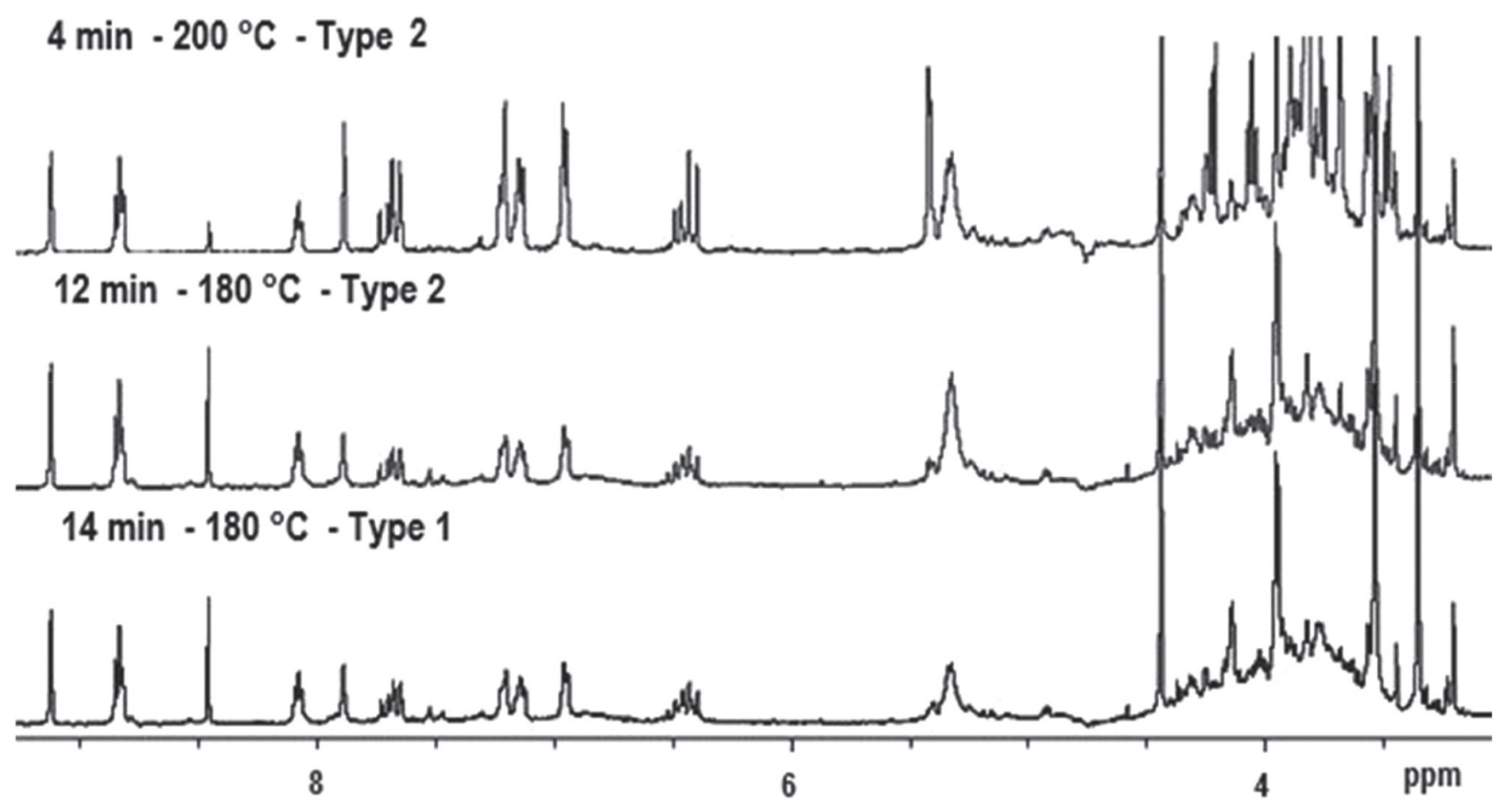

Figure 2. ${ }^{1} \mathrm{H}$ NMR spectra in ppm $\left(500 \mathrm{MHz}, \mathrm{D}_{2} \mathrm{O}\right)$ for different thermal conditions and types of arabica coffee. 


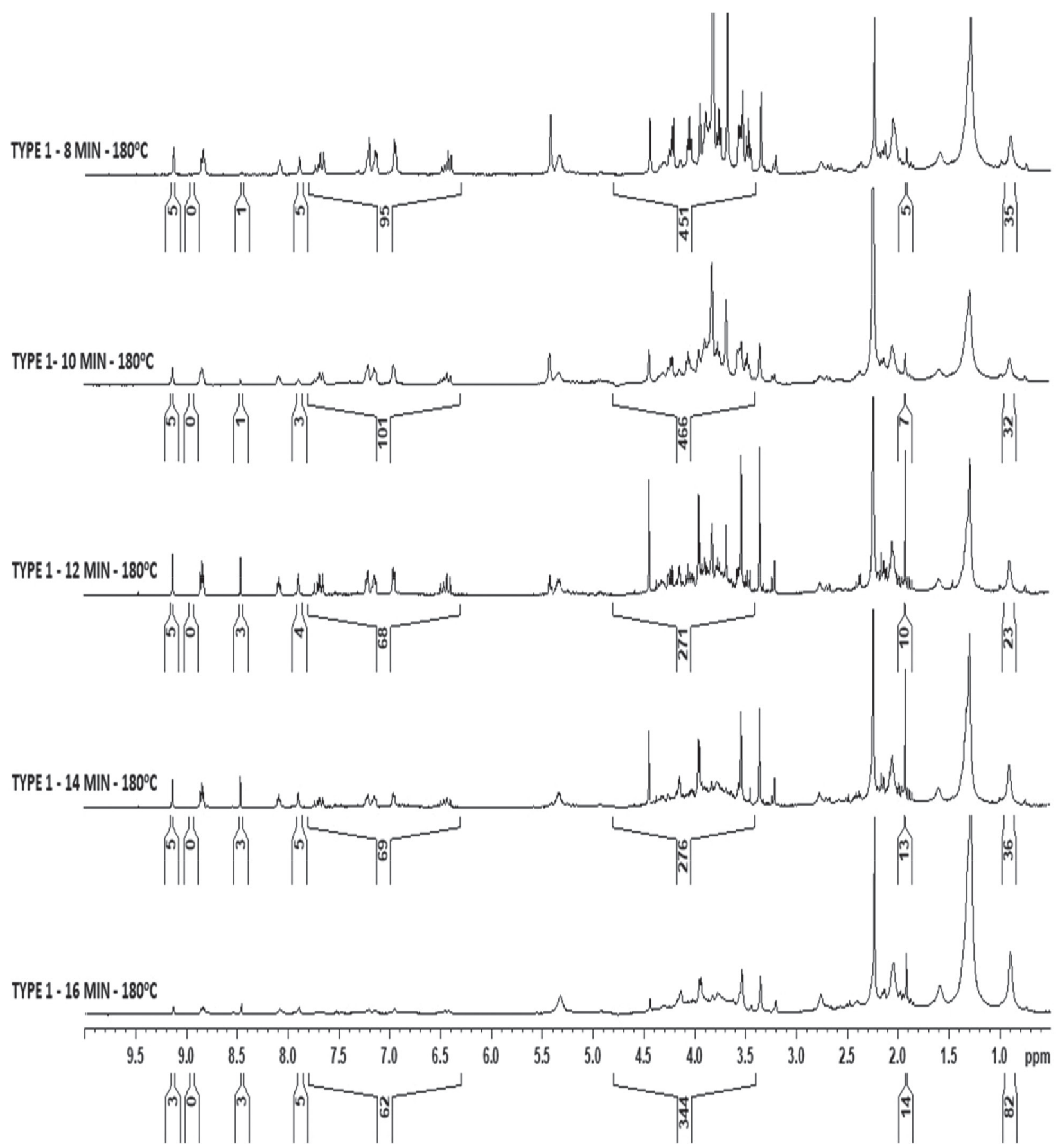

Figure 3. Spectra of ${ }^{1} \mathrm{H}$ NMR in ppm $\left(500 \mathrm{MHz}, \mathrm{D}_{2} \mathrm{O}\right)$ from samples $1-5$ of the roasting test-test 1.

signal integration. The compounds used for the evaluation were trigonelline (T), nicotinic acid (NA), formic acid (F), caffeine (C), chlorogenic acids (CQA), quinic acid (QA), fatty acids (FA), and carbohydrates (CHO), specifically.

This work used a semi-quantitative approach to analyze the transformations and main components involved. Thus, the integration values of the signals were directly proportional to the concentration of the components and indicated how the components were formed or consumed, regardless of a concentration unit. Through them, the slope of increase and decrease in the components found in this complex mixture were clear. We chose not to use concentration units to compare classes of compounds such as FA, CHO and CQA, because it would have been impossible to calculate the concentration as they did not have a fixed molar mass.

The decrease in the $\mathrm{CHO}$ integration values indicated their degradation during the roasting over time, therefore, their concentration values decreased dramatically after 10 min (Figure 4). The reductions in the values of $\mathrm{CHO}$ 

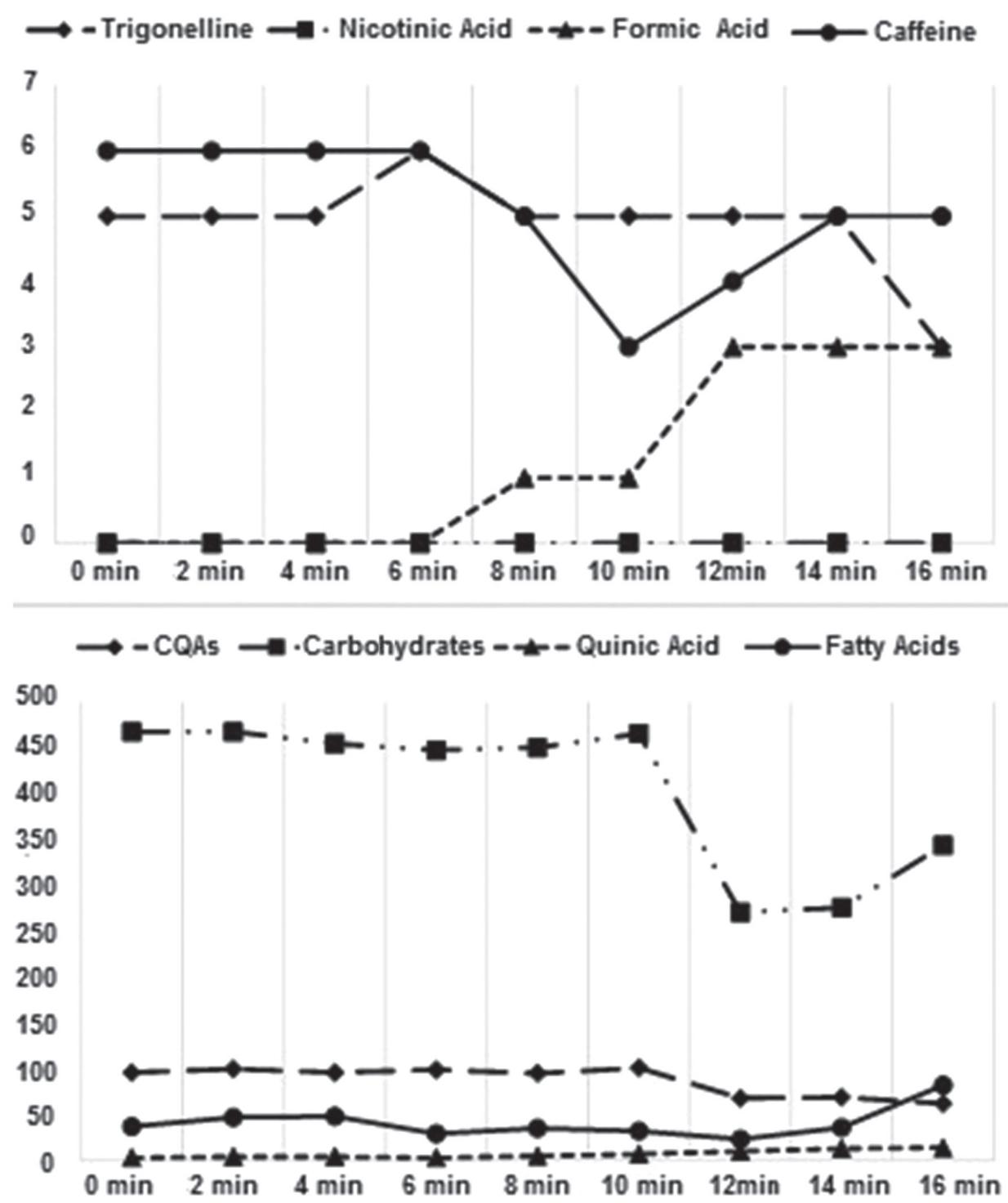

Figure 4. Line-graphs illustrating variation in the substances of interest according to the roasting time-test 1.

integration decreased even more sharply in type 2 coffee and with shorter heat exposure than type 1 coffee (Figure 5). As type 2 beans have a different quality and characteristic, they will behave differently in relation to the variation of temperature and time.

Figure 5 presents the results for test 2, which has different curves than test 1 (Figure 4). In both tests, only the FA, QA, and NA did not undergo changes in concentrations that can be substantially evidenced. This information is very useful to plan the roasting process, maintaining the properties of interest combined with the flavor and aroma. Another important issue is information about the characteristics of the bean to optimize the pyrolytic process. We observed that the lower bean quality decreased most compounds in just $4 \mathrm{~min}$ processing to a temperature of $180^{\circ} \mathrm{C}$. Thus, by varying the roasting conditions and the type of bean, the profile can be controlled better and less energy expended due to the shorter roasting time.

Figure 6 shows the spectra related to the structure of $\mathrm{C}$ at $7.75 \mathrm{ppm}$ for test 1 before and after the roasting procedure. Both spectra contain a clear and characteristic signal for that substance. However, the concentration of $\mathrm{C}$ was influenced by the time the sample was subjected to heating; the longer the time, the lower its concentration, which may vary by decomposition. Extended roasting times generate degradation products that interfere with the quality, aroma, and flavor of the drink. ${ }^{44}$

Figures 7 and 8 show data from test 3 , with less exposure time and higher temperature. The temperature increase to $200{ }^{\circ} \mathrm{C}$ anticipated the modification of the analyzed markers for the second minute of the process. This result was expected; however, the size of the alteration supported new 

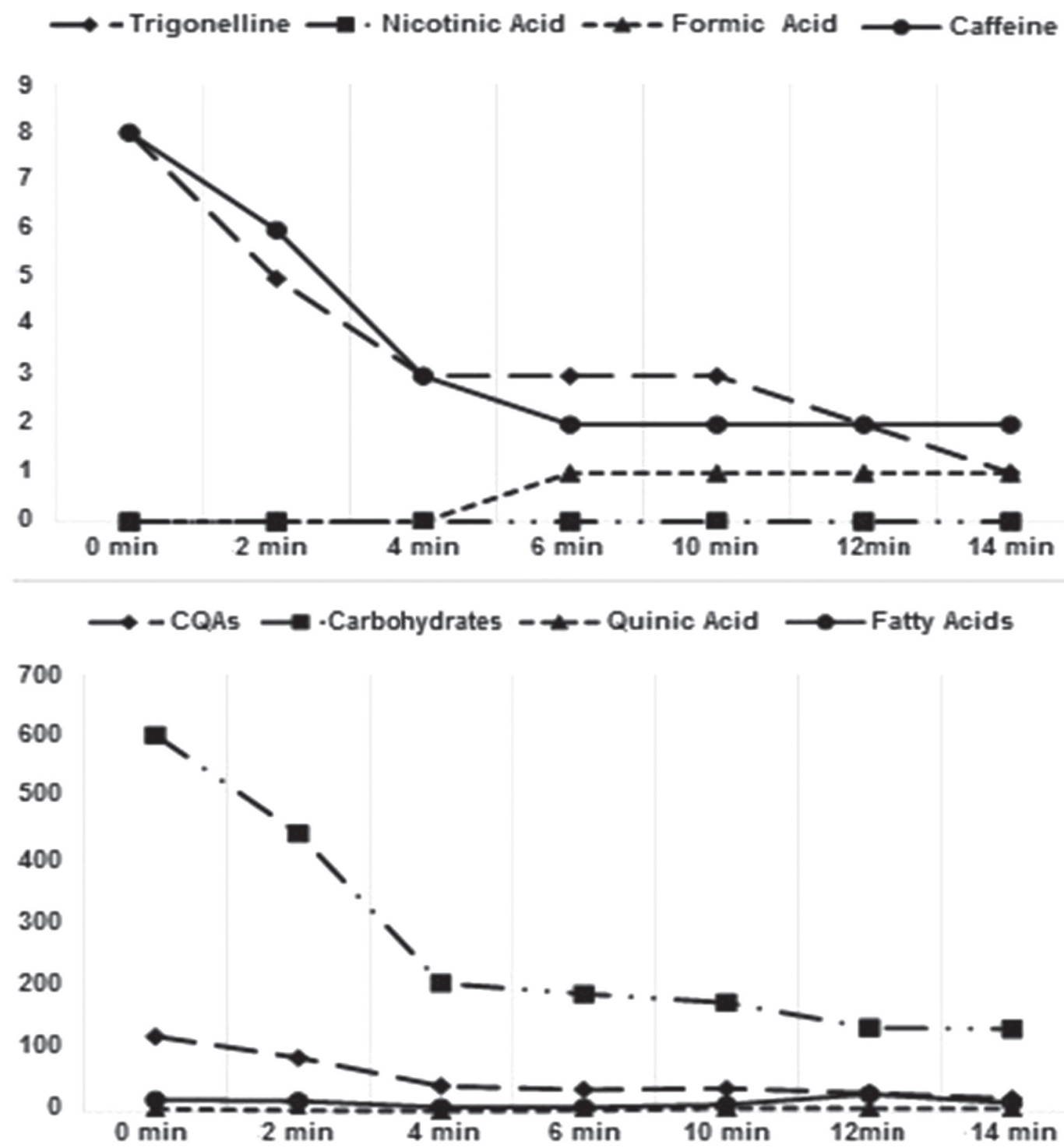

Figure 5. Line-graphs with variation of compounds according to the roasting time-test 2.
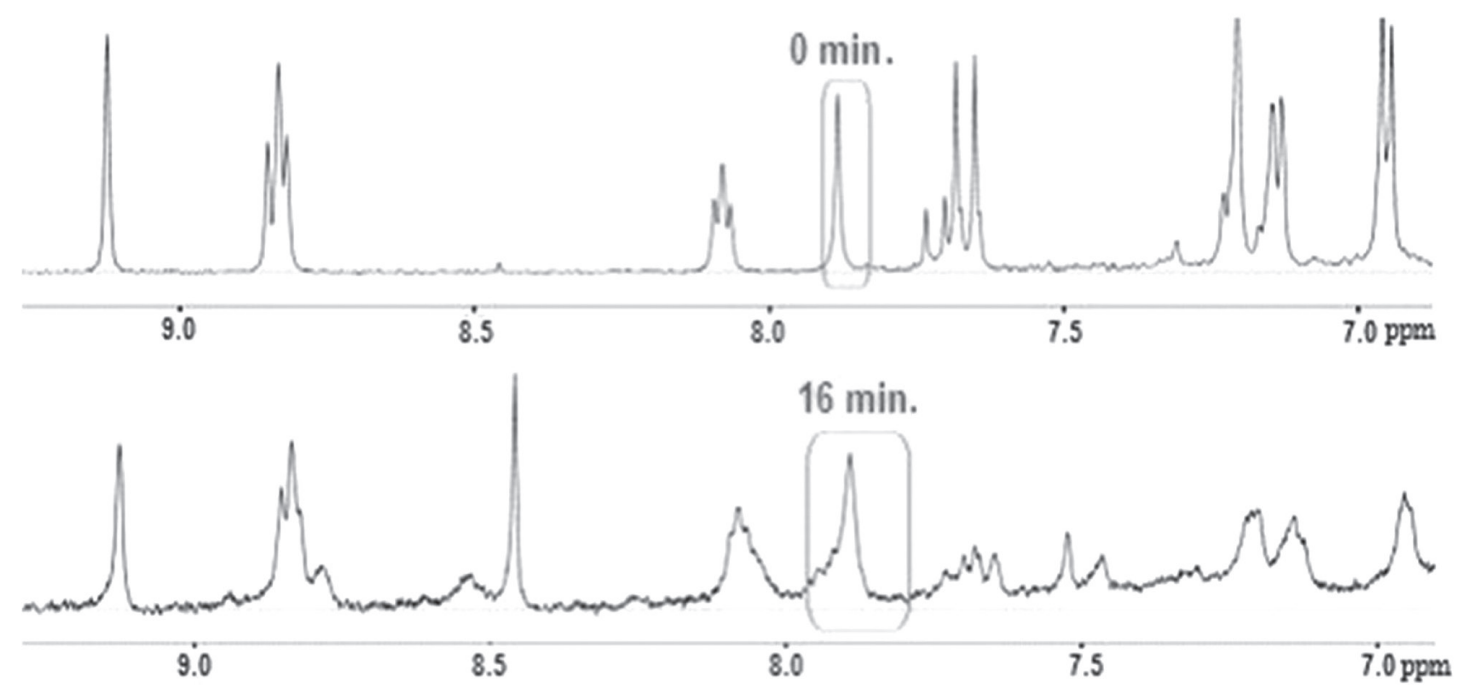

Figure 6. ${ }^{1} \mathrm{H}$ NMR spectrum in ppm $\left(500 \mathrm{MHz}, \mathrm{D}_{2} \mathrm{O}\right)$ before (above) and after (below) the roasting; emphasis on the sign (ppm) referring to caffeine. 


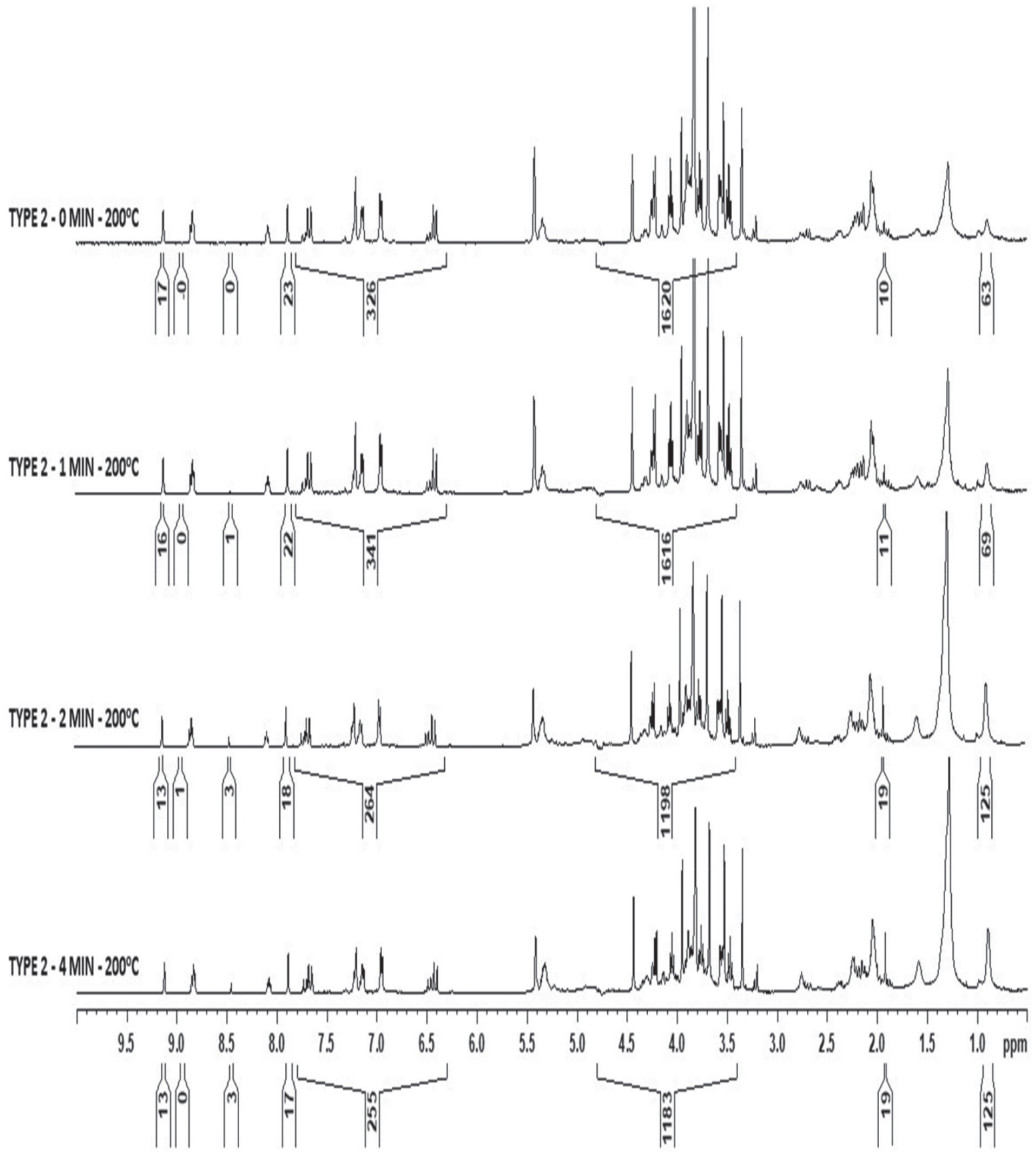

Figure 7. ${ }^{1} \mathrm{H}$ NMR spectra in ppm ( $\left.500 \mathrm{MHz}, \mathrm{D}_{2} \mathrm{O}\right)$ type 2 for test 3.

proposals for roasting optimization through quantitative data integrating the ${ }^{1} \mathrm{H}$ NMR signals. Remember that the signal area is directly proportional to the concentration of a group of molecules in a given spectral region.

In general, the FA concentrations varied little during the heating slope at the end of the three tests. The values fluctuated close to the initial values and signal overlap could have happened in the analysis. This could be due to the presence of unsaturated FA, which are more unstable than saturated FA contained in a lower concentration in coffee beans. In addition, saturated FA are more thermally stable and do not decompose at the temperatures used in the tests.

The CQA decreased in the three tests after heating, ${ }^{48}$ but they decayed at different times for each type of bean. In addition, the concentration of these acids underwent greater reduction in the lower quality beans. ${ }^{49}$ The QA increased concentration in longer roasting times (Figure 4). At low concentrations of some substances, they are better represented by line-graphs (Figures 4, 5 and 8 ) than spectral integrations (Figures 3, 6 and 7). 

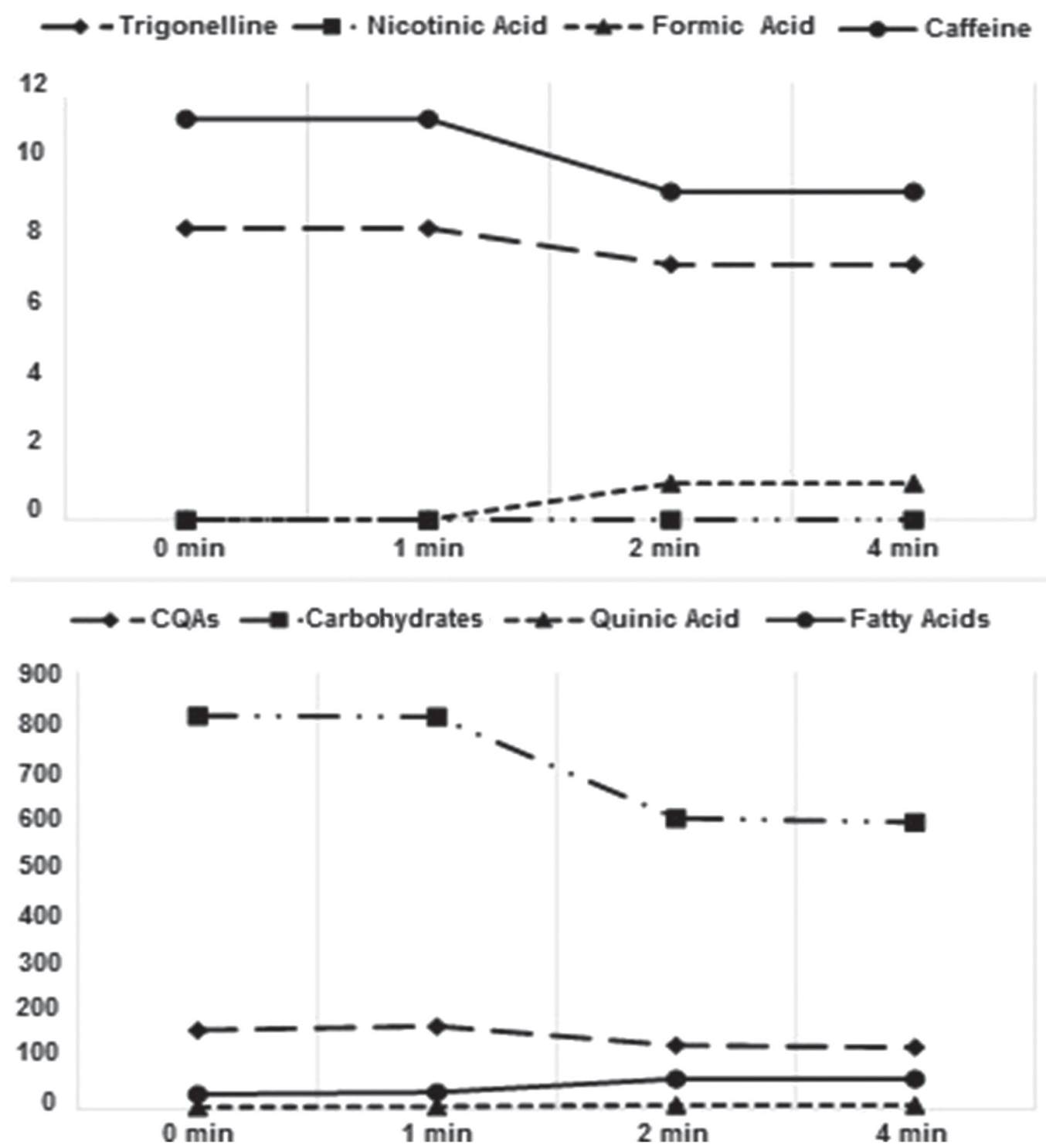

Figure 8. Line-graphs with the variation of the substances of interest according to the roasting time-test 3.

The concentration of $\mathrm{T}$ decreased rapidly in type 2 coffee (Figure 5) and slowly in type 1 (Figure 4). The concentration of this component is directly influenced by both the quality of the bean and the roasting time..$^{50}$

The NA was formed only in test 2 with 14 min of exposure although its integration was rounded to zero. The concentration of NA may be related both to the quality of the bean and roasting time. In addition, the FA is an indication of the roasting time and is linearly related to prolonged exposure of thermal energy.

C decreased strongly in type 2 (Figure 5), and less markedly in type 1 coffee (Figure 4). Considering the importance of the essential human health components found in coffee beans, as well as the roasting time, energy demand, and processing costs, the proposal was to reduce the roasting time (type $2,4 \mathrm{~min}, 200^{\circ} \mathrm{C}$ ) if proven efficient, guaranteeing quality without losing the natural organoleptic properties of coffee. The concentrations of $\mathrm{C}$ and $\mathrm{T}$ can also be used to identify the types of coffee species contained in the analyzed matrix, such as: arabica, robusta, or liberica. ${ }^{51}$

\section{Commercial coffees}

Analysis of the coffees sold in Goiânia found similar spectral profiles, demonstrating the proximity in the quality of the raw materials and coffee species. In addition, the roasting process can be seen for each type analyzed, according to the presence of compounds present with the longest roasting time. However, there was noticeable lack of standardization in the component profile according to the classification of traditional coffee, extra strong, dark roast (Figures 9 and 10). 


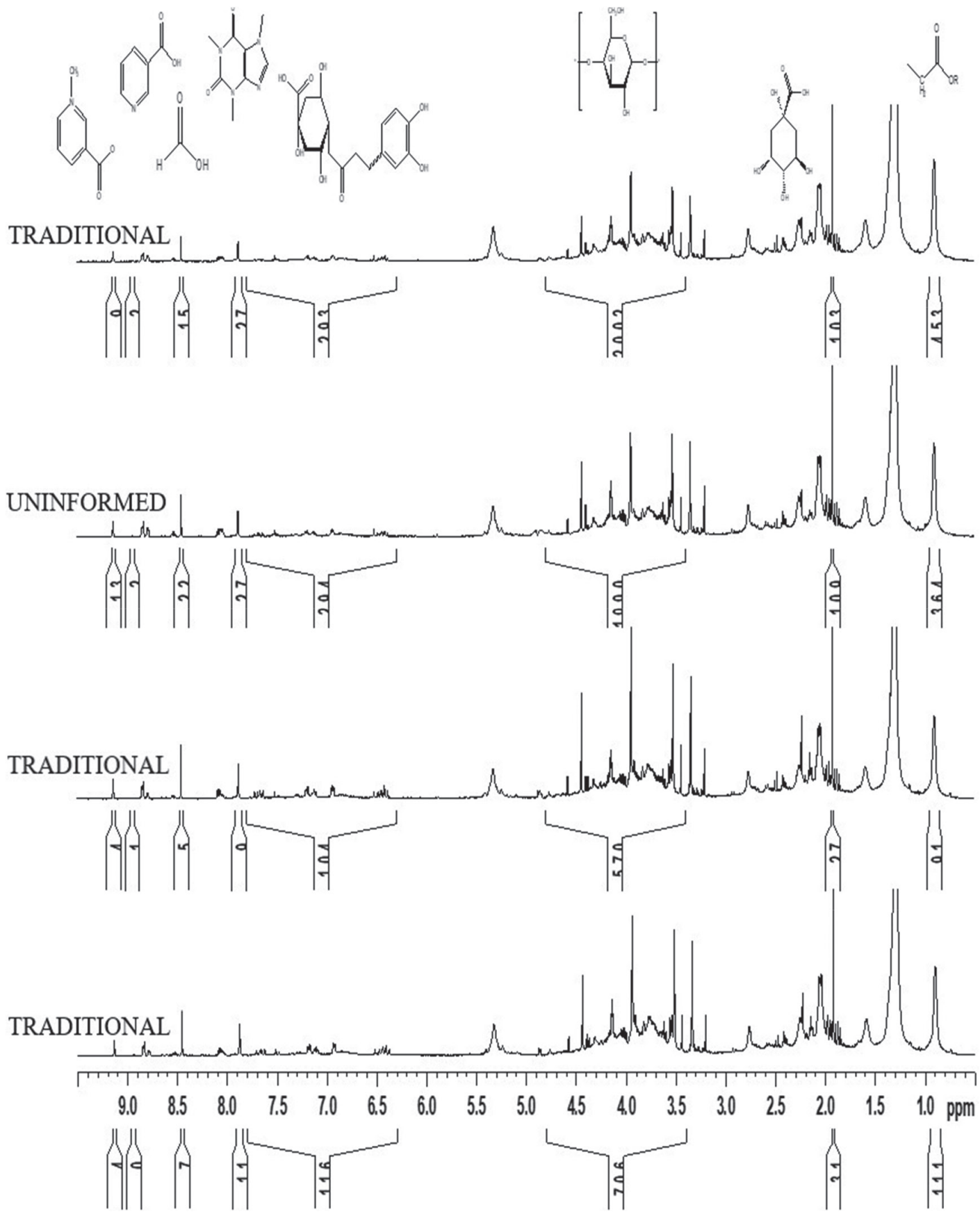

Figure 9. ${ }^{\mathrm{H}} \mathrm{H}$ NR spectra in ppm $\left(500 \mathrm{MHz}, \mathrm{D}_{2} \mathrm{O}\right)$ of commercial samples $1,2,3$, and 4 (from bottom to top), with the respective chemical structures of the identified compounds (top).

Only traditional coffees did not present the singlet signal at $8.65 \mathrm{ppm}$ of $\mathrm{F}$. This signal is indicative of the roasting time, as it is obtained by degradation caused by the prolonged heating time.

The CHO signals varied near 4.40 ppm mainly in samples 3, 4, and 7, changing the proportion between them. Only sample 9 exhibited a signal at $3.04 \mathrm{ppm}$. As this signal is not common in any roasting time, it may indicate a presence of impurity or strange body to the coffee. The general impression of commercial products is that their chemical profiles are similar to the coffees generated with a longer roasting time and exhibit a noticeable change in the $\mathrm{CHO}$ profile.

\section{Chemometric analyzes}

Figure 11 presents the graph of PCA scores, with a separation of the samples according to the roasting time that they were submitted. The negative scores of the first main component (PC1) are for the samples that were roasted for 


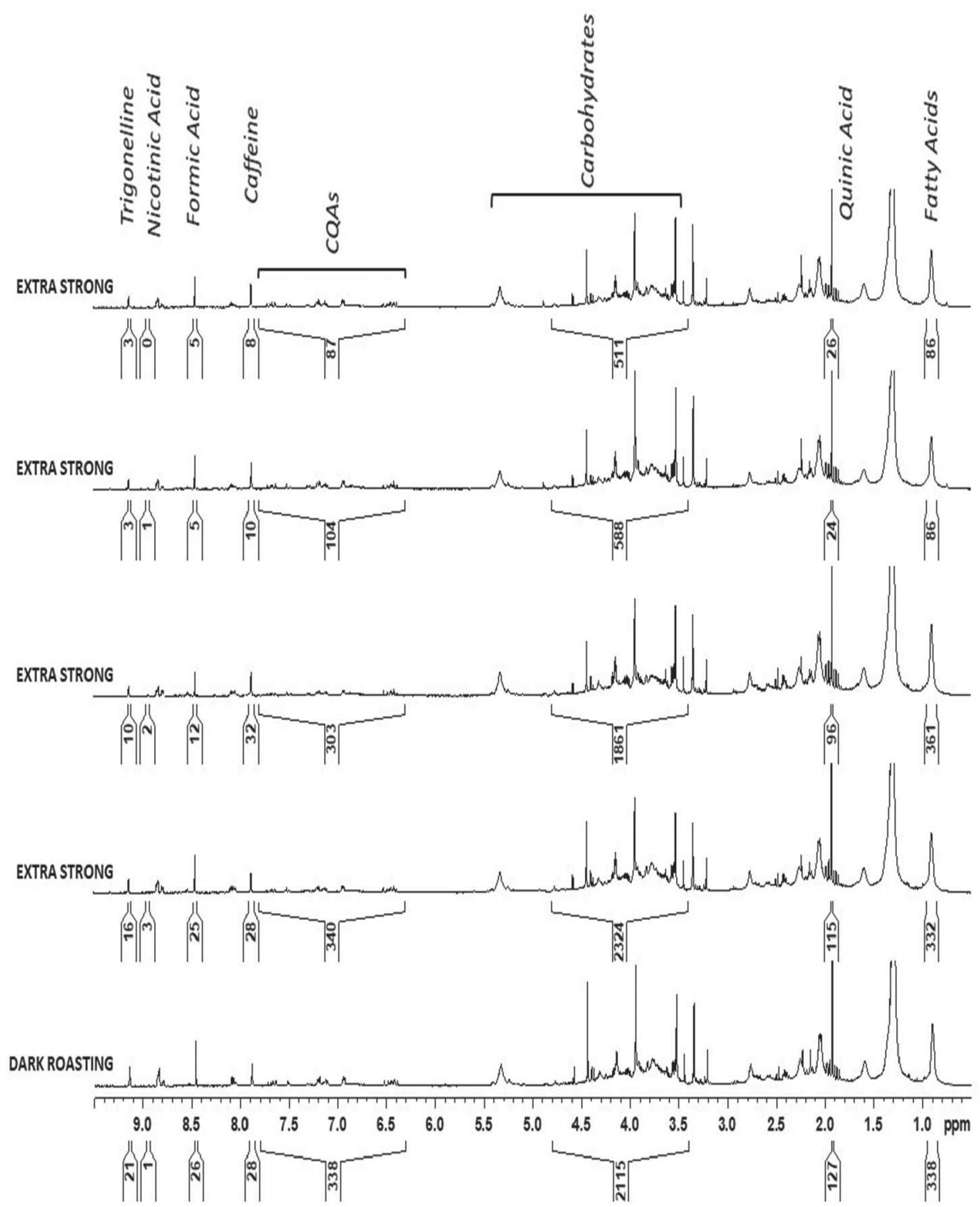

Figure 10. ${ }^{1} \mathrm{H}$ NMR spectra in ppm $\left(500 \mathrm{MHz}, \mathrm{D}_{2} \mathrm{O}\right)$ of commercial samples 5, 6, 7, 8, and 9 (from bottom to top), with the respective names of the identified compounds (top).

longer, and the positive scores are for the other samples, which had a more preserved chemical profile due to a shorter heat exposure. Thus, samples with a predominantly natural profile differed from those that had a greater number of compounds generated by the roasting process. The greatest contribution to the explanatory variance could be seen up to the fourth main component, with the total accumulated value of $74.3 \%$. After that, the increment became smaller and smaller, indicating that most of the information was preserved in the first components ( $\mathrm{PC} 1$ and PC2). This can be explained mainly by the signals of major compounds that result in a spectrum of medium complexity.

Sample 4 (commercial coffee) was most influenced in the PCA graph, with the highest values plotted in the influence graph. On the other hand, the $\mathrm{CHO}$ region presented the variables responsible for the dispersion of the samples along PC1, predominantly identified in the loadings graph. This finding can be explained by the large 


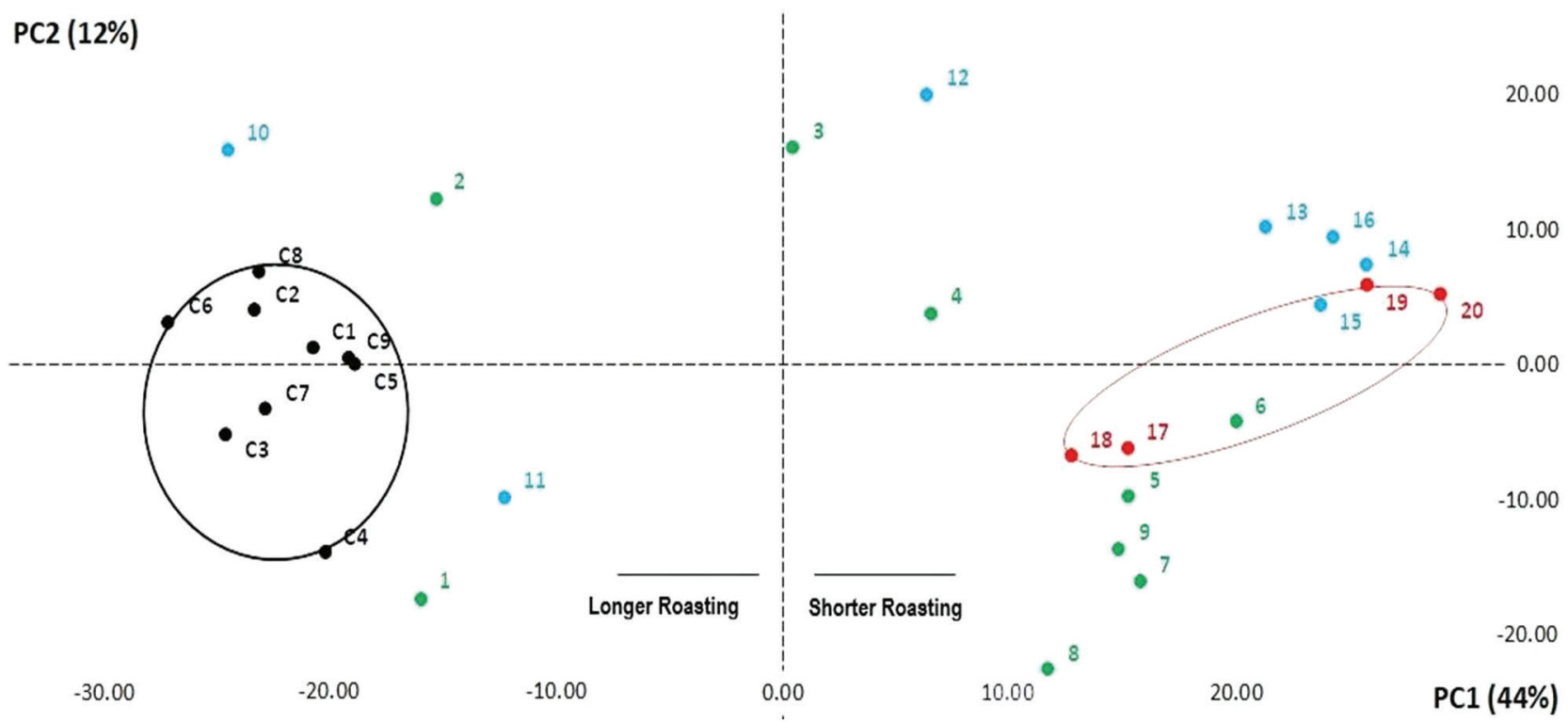

Figure 11. Graph of PCA scores generated with the samples from the roasting process (green: test 1; blue: test 2; red: test 3; black: commercial coffees).

change in the $\mathrm{CHO}$ profile with the roasting time of $10 \mathrm{~min}$ for the highest quality roasted beans (type 1).

The commercial samples were grouped close to the samples with longer roasting time made at EA/UFG. In the second main component (PC2), the samples with the longest roasting time differ in positive values, especially those that were also found in negative PC1 values. The samples with longer roasting time $(1,2,3,10$, and 11) exhibited a product profile most accepted by Brazilian consumers. ${ }^{46}$

Test 3 presented a profile similar to that of most preserved because it has a shorter roasting time, grouping through PCA with the samples 6, 7, 9-test 1 and 14, 15, 16-test 2, despite the use of temperature above $200{ }^{\circ} \mathrm{C}$. Evaluation of the chemical profile indicates that different strategies can be used to preserve some components without significantly changing the sensory characteristics of coffee. Figure 2 shows this possibility of preserving the $\mathrm{CHO}$ profile (region between 3.01 and 4.80) in a sample from test 3 , and still presenting a very similar profile in the other regions of the spectrum.

The ${ }^{1} \mathrm{H}$ NMR technique used efficiently evaluated the quality of the sample and the characteristics that coffee presents in different roasting times. This technique can also be used to identify possible adulterants in the drink, effectively replacing other analytical techniques, such as molecular biology methods based on deoxyribonucleic acid (DNA), ${ }^{52}$ capillary electrophoresis (CE),${ }^{53}$ HPLC, ${ }^{54}$ and GC-MS, ${ }^{55}$ which require longer analysis time and larger quantity of consumables per test. In addition, ${ }^{1} \mathrm{H}$ NMR is less expensive and produces less residues in sample preparation.

\section{Conclusions}

${ }^{1} \mathrm{H}$ NMR proved to be able to monitor the roasting process, qualifying the intact bean and the chemical profile of the coffee without the need for pre-treatment and derivatization, according to the different physical conditions used, such as the roasting time and temperature. This analysis was able to identify the appearance of compounds from adulteration that are normally inserted into the product to reduce the production cost, which drop in the quality and nutritional capacity of the drink, in addition to controlling the appearance of unwanted compounds.

This studied highlighted the change in the roasting profile according to the quality of the bean. This evaluation was subjective, made by a coffee master, and compared with other published works. ${ }^{39}$ A completely different product was found for the two types of coffee evaluated. Furthermore, the proposal to guarantee the product quality through a drastic reduction in the roasting period (type 2 , 4 min, $200{ }^{\circ} \mathrm{C}$ ) was guaranteed, since the chemical profile of the main components of interest was maintained without suffering degradation and without the loss of the organoleptic properties of coffee beans. Another factor was to identify quality as a determining variable in the degradation of genuine coffee compounds, with this behavior expected only for a rise in temperature. Commercial coffees exhibited good similarity with coffees roasted at EA/UFG, changing only the concentrations between their components. The commercial products with longer roasting time were most similar to our roasted coffees. The convergence of the signs and assigning authenticity to them could help detect adulterants, due to the high similarity with authentic arabica coffee beans that 
were only ground and roasted. In addition, there is a need to monitor certain components for a better nutritional profile through a simple and quick analysis, as is the case with ${ }^{1} \mathrm{H}$ NMR compared to other analytical techniques, which can be easily applied to other organic matrices similar to coffee beans.

\section{Acknowledgments}

The authors would like to thank CNPq (National Council for Scientific and Technological Development) and CAPES (Coordination for the Improvement of Higher Education Personnel) for their fellowships and financial support; to the UFG School of Agronomy for sample preparation and assistance in the roasting stages.

\section{References}

1. Nabais, J. V.; Carrott, P.; Carrott, M. R.; Luz, V.; Ortiz, A. L.; Bioresour. Technol. 2007, 99, 7224.

2. dos Santos, D. C.; Adebayo, M. A.; Lima, E. C.; Pereira, S. F. P.; Cataluña, R.; Saucier, C.; Thue, P. S.; Machado, F. M.; J. Braz. Chem. Soc. 2015, 26, 924.

3. Bhumiratana, N.; Adhikari, K.; Chambers, E.; Sci. Technol. 2011, 44, 2185.

4. N'Diaye, A.; Poncet, V.; Louarn, J.; Hamon, S.; Noirot, M.; Plant Syst. Evol. 2005, 253, 95.

5. http://www.epamig.ufla.br/geosolos/publicacoes/2011/3.pdf, accessed in July 2020.

6. Pereira, G. V. M.; Soccol, V. T.; Brar, S. K.; Neto, E.; Soccol, C. R.; Crit. Rev. Food Sci. Nutr. 2017, 57, 2775.

7. Tran, H. T.; Vargas, C. A. C.; Lee, L. S.; Furtado, A.; Smyth, H.; Henry, R.; Tree Genet. Genomes 2017, 13, 54.

8. Huch, M.; Franz, C. M. A. P. In Advances in Fermented Foods and Beverages Improving Quality, Technologies and Health Benefits; Holzapfel, W., ed.; Woodhead Publishing: Sawston, 2015, p. 501.

9. Badolato, E. S. G.; Martins, M. S.; Aued-Pimentel, S.; Alaburda, J.; Kumagai, E. E.; Baptista, G. G.; Rosenthal, A.; J. Braz. Chem. Soc. 2006, 17, 989.

10. Perrone, D.; Donangelo, C. M.; Farah, A.; Food Chem. 2008, 110, 1030.

11. Licciardi, R.; Pereira, R. G. F. A.; Mendonça, L. M. V. L.; Furtado, E. F.; Cienc. Tecnol. Aliment. 2015, 25, 425.

12. Ky, C.-L.; Louarn, J.; Dussert, S.; Guyot, B.; Hamon, S.; Noirot, M.; Food Chem. 2001, 75, 223

13. Baeza, G.; Amigo-Benavent, M.; Sarriá, B.; Goya, L.; Mateos, R.; Bravo, L.; Food Res. Int. 2014, 62, 1038.

14. Yeomans, M.; Chemosens. Percept. 2010, 3, 34.

15. Neto, E. M. C.; Resende, J. J.; Acta Sci., Biol. Sci. 2004, 26, 143.
16. Sato, Y.; Itagaki, S.; Kurokawa, T.; Ogura, J.; Kobayashi, M.; Hirano, T.; Int. J. Pharm. 2011, 403, 136.

17. Wang, H.-Y.; Qian, H. H.; Yao, W.-R.; Food Chem. 2011, 128, 573.

18. Araujo, F. A.; Mancini-Filho, J.; Hig. Aliment. 2006, 20, 60.

19. Sanz, C.; Czerny, M.; Cid, C.; Schieberle, P.; Eur. Food Res. Technol. 2002, 214, 299.

20. Schenker, S.; Heinemann, C.; Huber, M.; Pompizzi, R.; Perren, R.; Escher, F.; J. Food Sci. 2002, 67, 60.

21. Escher, F.; Baggenstoss, J.; Poisson, L.; Kaegi, R.; Perren, R.; J. Agric. Food Chem. 2008, 56, 5836.

22. Bouhsain, Z.; Garrigues, J. M.; Garrigues, S.; Guardia, M.; Vib. Spectrosc. 1999, 21, 143.

23. Briandet, R.; Kemsley, E. K.; Wilson, R. H.; J. Agric. Food Chem. 1996, 44, 170.

24. Esteban-Díez, I.; González, S. J. M.; Pizarro, C.; Anal. Chim. Acta 2004, 514, 57.

25. Martin, M. J.; Pablos, F.; González, A. G.; Talanta 1998, 46, 1259.

26. del Castillo, M. L. R.; Herraiz, M.; Blanch, G.; J. Agric. Food Chem. 1999, 47, 1525.

27. Carrera, F.; León-Camacho, M.; Pablos, F.; González, A. G.; Anal Chim. Acta 1998, 370, 131.

28. Jham, G. N.; Berhow, M. A.; Manthey, L. K.; Palmquist, D. A.; Vaughn, S. F.; J. Braz. Chem. Soc. 2008, 19, 1462.

29. Dias, R. C. E.; Campanha, F. G.; Vieira, L. G. E.; Ferreira, L. P.; Pot, D.; Marraccini, P.; Benassi, M. T.; J. Agric. Food Chem. 2010, 58, 88 .

30. Romano, R.; Santini, A.; le Grottaglie, L.; Manzo, N.; Visconti, A.; Ritieni, A.; J. Food Compos. Anal. 2014, 35, 1.

31. di Bella, G.; Potorti, A. G.; lo Turco, V.; Saitta, M.; Dugo, G.; Food Control 2014, 41, 185.

32. Bosco, M.; Toffanin, R.; de Palo, D.; Zatti, L.; Segre, A.; J. Sci. Food Agric. 1999, 79, 869.

33. Tanokura, M.; Wei, F.; Furihata, K.; Koda, M.; Hu, F.; Myakawa, T.; J. Agric. Food Chem. 2012, 60, 1005.

34. Charlton, A. J.; Farrington, W. H. H.; Brereton, P.; J. Agric. Food Chem. 2002, 50, 3098.

35. Zambonin, C. G.; Balest, L.; de Benedetto, G. E.; Palmisano, F.; Talanta 2005, 66, 261.

36. Risticevic, S.; Carasek, E.; Pawliszyn, J.; Anal. Chim. Acta 2008, 617, 72 .

37. Bessa, C. D. P. B.; de Andrade, J. P.; de Oliveira, R. S.; Domingos, E.; Santos, H.; Romão, W.; Bastida, J.; Borges, W. S.; J. Braz. Chem. Soc. 2017, 28, 819.

38. Gorder, R. A. V.; Fadai, N. T.; Melrose, J.; Please, C. P.; Schulman, A.; Int. J. Heat Mass Transfer 2017, 104, 787.

39. Monakhova, Y. B.; Ruge, W.; Kuballa, T.; Ilse, M.; Winkelmann, O.; Diehl, B.; Thomas, F.; Lachenmeier, D. W.; Food Chem. 2015, 182, 178. 
40. Kemsley, E. K.; Defernez, M.; When, E.; Watson, A. D.; Gunning, Y.; Colquhoun, I. J.; le Gall, G.; Williamson, D.; Food Chem. 2017, 216, 106.

41. Gunning, Y.; Defernez, M.; Watson, A. D.; Beadman, N.; Colquhoun, I. J.; le Gall, G.; Philo, M.; Garwood, H.; Williamson, D.; Davis, A. P.; Kemsley, E. K.; Food Chem. 2018, $248,52$.

42. Lachenmeier, D. W.; Schwarz, S.; Teipel, J.; Hegmanns, M.; Kuballa, T.; Walch, S. G.; Breitling-Utzmann, C. M.; Toxics 2019, 7, DOI 10.3390/toxics7010001.

43. Mottram, D. S.; Wedzicha, B. L.; Dodson, A. T.; Nature 2002 , $419,448$.

44. de Souza, R. M. N.; Benassi, M. T.; J. Braz. Chem. Soc. 2012, $23,1347$.

45. Howell, G.; SCAA Universal Cupping Form and How to Use $i t ; 10^{\text {th }}$ Annual Conference and Exhibition Peak of Perfection: Denver, USA, 1998.

46. http://www.abic.com.br/certificacao/qualidade/categorias-dequalidade, accessed in July 2020.

47. Ferreira, A. G.; Tavares, L. A.; Quim. Nova 2006, 29, 911.
48. Vignoli, J. A.; Bassoli, D. G.; Benassi, M. T.; Food Chem. 2011, $124,863$.

49. Monteiro, M. C.; Trugo, L. C.; Quim. Nova 2005, 28, 637.

50. Morais, S. A. L.; Aquino, F. J. T.; Nascimento, P. M. N.; Nascimento, E. A.; Chang, R.; Quim. Nova 2009, 32, 327.

51. Berthou, F.; Mathiel, C.; Vedel, F.; Theor. Appl. Genet. 1983, $65,77$.

52. Combes, M. C.; Joët, T.; Lashermes, P.; Food Control. 2018, $88,198$.

53. Daniel, D.; Lopes, F. S.; dos Santos, V. B.; do Lago, C. L.; Food Chem. 2018, 243, 305.

54. Domingues, D. S.; Pauli, E. D.; de Abreu, J. E.; Massura, F. W.; Cristiano, V.; Santos, M. J.; Nixdorf, S. L.; Food Chem. 2014, 146, 353.

55. Pauli, E. D.; Barbieri, F.; Garcia, P. S.; Madeira, T. B.; Acquaro, V. R.; Scarminio, I. S.; Nixdorf, S. L.; Food Res. Int. 2014, 61, 112.

Submitted: April 16, 2020

Published online: August 10, 2020 